\title{
Análise de idosos ambulatoriais quanto ao estado nutricional, sarcopenia, função renal e densidade óssea
}

\author{
Analysis of elderly outpatients in relation to nutritional \\ status, sarcopenia, renal function, and bone density
}

Franciany Viana Salmaso', Patrícia dos Santos Vigário², Laura Maria Carvalho de Mendonça ${ }^{3}$, Miguel Madeira', Leonardo Vieira Netto', Marcela Rodrigues Moreira Guimarães ${ }^{4}$, Maria Lucia Fleiuss de Farias ${ }^{1}$

\footnotetext{
' Disciplina de Endocrinologia da Faculdade de Medicina, Universidade Federal do Rio de Janeiro (UFRJ), Rio de Janeiro, RJ, Brasil

2 Programa de Pós-Graduação em Ciências da Reabilitação Unisuam, Rio de Janeiro, RJ, Brasil ${ }^{3}$ Disciplina de Reumatologia da Faculdade de Medicina, UFRJ Rio de Janeiro, RJ, Brasil ${ }^{4}$ Programa de Pós-Graduação em Neurologia, UFRJ, Rio de Janeiro, RJ, Brasil
}

\section{RESUMO}

Objetivo: Avaliar relações entre estado nutricional, sarcopenia e osteoporose em idosas. Sujeitos e métodos: Estudamos 44 mulheres, 67-94 anos, mediante miniavaliação nutricional (MAN), filtração glomerular (FG) corr. 1,73 $\mathrm{m}^{2}$, índice de massa corporal (IMC), circunferência da panturrilha e braquial (CP e CB), densidade mineral óssea e composição corporal, DXA (massa gorda - MG; massa magra - MM). Aferimos sarcopenia: IMM = MM MSS + MIS/altura². Utilizamos o coeficiente de correlação de Pearson, e $p<0,05$ como significativo. Resultados: MNA e IMM se correlacionaram positivamente com IMC, CP, CB e MG. A idade influenciou negativamente FG corr., IMC, MG, IMM e CP. Quatorze tinham história de fraturas osteoporóticas. $\mathrm{O}$ mais baixo T-score foi diretamente relacionado a MAN e MG. Conclusões: $\mathrm{O}$ envelhecimento acarretou o declínio da FG, MG e massa muscular; a circunferência da panturrilha e braquial refletiu estado nutricional e composição corporal; e as grandes influências na DMO foram estado nutricional e MG. Arq Bras Endocrinol Metab. 2014;58(3):226-31

Descritores

Nutrição; sarcopenia; densidade mineral óssea; osteoporose; envelhecimento

\section{ABSTRACT}

Objectives: To evaluate relationships between nutritional status, sarcopenia and osteoporosis in older women. Subjects and methods: We studied 44 women, 67-94 years, by mini-nutritional assessment (MAN), glomerular filtration corr. $1.73 \mathrm{~m}^{2}$, body mass index (BMI), arm circumference and calf (CP and $\mathrm{CB}$ ), bone mineral density and body composition, DXA (fat mass MG; lean MM). We gauge sarcopenia: IMM MM = MSS + MIS/height ${ }^{2}$. We used the Pearson correlation coefficient, $\mathrm{p}<0.05$ as significant. Results: MNA and IMM were positively correlated with $\mathrm{BMI}, \mathrm{CP}, \mathrm{CB}$ and MG. Age influenced negatively FG corr., BMI, FM, IMM and CP. Fourteen had a history of osteoporotic fractures. The lowest T-score was directly related to MAN and MG. Conclusions: The aging caused the decline of FG, fat mass and muscle; the calf circumference, and brachial reflected nutritional status and body composition; and major influences on BMD were nutritional status and fat mass.Arq Bras Endocrinol Metab. 2014;58(3):226-31

\section{Keywords}

Nutrition; sarcopenia; bone mineral density; osteoporosis; aging 


\section{INTRODUÇÃO}

$\mathrm{E}$ stima-se que até 2025 o Brasil alcançará a posição do sexto país do mundo em número de idosos (1). Com o envelhecimento, surgem as doenças crônicas degenerativas, a reabilitação tardia e os sinais de doença em fases avançadas, comprometendo, de forma geral, a funcionalidade e a qualidade de vida do idoso. Nesse processo, também há redução da massa e da força muscular, fadiga, alteração da marcha e do equilíbrio, perda de apetite e consequente redução do peso. A desnutrição contribui para a insuficiência de diversos órgãos, inclusive renal (2). Nosso objetivo é avaliar as relações entre o estado nutricional, sarcopenia e função renal com a densidade mineral óssea em idosos com mais de 65 anos.

\section{SUJEITOS E MÉTODOS}

O presente estudo faz parte de um protocolo de pesquisa em idosos acompanhados no ambulatório de Geriatria do Hospital Universitário Clementino Fraga Filho da Universidade Federal do Rio de Janeiro (HUCFF-UFRJ), uma população clinicamente estável e que não necessita de acompanhamento específico em outros ambulatórios de especialidade. O estudo foi aprovado pelo CEP em 25/2/2010 e todas as participantes assinaram o Termo de Consentimento Livre e Esclarecido (TCLE).

Dentre as mulheres idosas com idade $\geq 65$ anos do ambulatório, capazes de responder aos questionamentos sobre consumo alimentar e deambular sem auxílio, os critérios de exclusão foram: ser cadeirante ou deambular com dificuldade, apresentar neoplasia ativa, estar em diálise, possuir doença crônica consumptiva ou em fase avançada e evidente déficit cognitivo. Eram aceitas idosas com discreta deficiência cognitiva desde que o(a) acompanhante fosse capaz de prestar as informações necessárias. O estudo foi iniciado com 59 pacientes, mas somente 44 concluíram a avaliação.

Foram buscados nos prontuários dados referentes a comorbidades e medicamentos usados de forma crônica e resultados de exames de sangue de rotina realizados nos últimos 4 meses, periodicidade usual de atendimento no Ambulatório de Geriatria, incluindo cálcio e PTH (quimioluminescência, Immulite 2000 - Siemens Healthcare Diagnostics Ltda., EUA - valores de normalidade $12-65 \mathrm{pg} / \mathrm{ml}$ ). A taxa de filtração glomerular (FG) foi obtida por meio da Equação
Cockroft Gault (3), sendo corrigida para 1,73 $\mathrm{m}^{2}$ para aplicarmos a classificação de doença renal crônica definida pelo K/DOQI 2002 (4): fase 3A (FG 45-59 ml/ $\left.\mathrm{min} / \mathrm{l}, 73 \mathrm{~m}^{2}\right)$; fase $3 \mathrm{~B}\left(\mathrm{FG} 30-44 \mathrm{ml} / \mathrm{min} / \mathrm{l}, 73 \mathrm{~m}^{2}\right)$; fase $4\left(\mathrm{FG} 15-29 \mathrm{ml} / \mathrm{min} / \mathrm{l}, 73 \mathrm{~m}^{2}\right)$ e fase $5(\mathrm{FG}<15$ $\left.\mathrm{ml} / \mathrm{min} / 1,73 \mathrm{~m}^{2}\right)$.

As pacientes foram pesadas e medidas pelo mesmo examinador, em balança digital Filizola com capacidade $150 \mathrm{~kg}$, descalças e usando roupas leves. Calculado $\mathrm{o}$ índice de massa corporal $(\mathrm{IMC}=$ peso $(\mathrm{kg}) /$ estatura $(\mathrm{m})^{2}$, utilizamos os critérios de Nutrition Screening Initiative - NSI 2000: magreza ou baixo peso (IMC $<22 \mathrm{~kg} / \mathrm{m}^{2}$ ); eutrofia (IMC $22 \mathrm{a}<27 \mathrm{~kg} / \mathrm{m}^{2}$ ); sobrepeso (IMC $27 \mathrm{a}<30 \mathrm{~kg} / \mathrm{m}^{2}$ ) e obesidade (IMC $\geq$ $\left.30 \mathrm{~kg} / \mathrm{m}^{2}\right)(5)$. Com fita metálica flexível Sanny, medimos a circunferência do braço direito $(\mathrm{CB}$, no ponto médio entre o acrômio e o olécrano, com braços relaxados) e a circunferência da panturrilha $(\mathrm{CP}$, com a idosa sentada, joelho flexionado formando um ângulo de noventa graus com o chão; foi considerado o ponto de maior circunferência da panturrilha da perna direita). $\mathrm{CB}<22 \mathrm{~cm}$ e $\mathrm{CP}<31 \mathrm{~cm}$ são compatíveis com desnutrição segundo o questionário $\operatorname{MAN}(6)$.

$\mathrm{O}$ risco nutricional foi aferido pelo MAN, que contempla avaliação antropométrica, questionário alimentar, avaliação global e autoavaliação do idoso. Caso a idosa tivesse dúvidas, $\mathrm{o}(\mathrm{a})$ respectivo(a) acompanhante relatava seu consumo alimentar. O MAN é dividido $\mathrm{em}$ : triagem e avaliação global. Constam da triagem informações sobre diminuição da ingestão alimentar por perda de apetite, problemas digestivos, dificuldade para mastigar ou deglutir, perda de peso nos últimos meses, mobilidade, estresse psicológico ou doença aguda nos últimos três meses e problemas neuropsicológicos (demência ou depressão); o escore máximo da triagem é de 14 pontos e pontuações $\leq 11$ sugerem desnutrição. A avaliação global inclui questionário alimentar, autoavaliação do idoso e avaliação antropométrica (circunferência do braço e da panturrilha). O escore máximo da avaliação global é de 16 pontos. Dessa forma, o MAN totaliza uma pontuação máxima de 30 pontos; pontuações de 23,5 a 17 indicam risco nutricional e abaixo de 17 indicam desnutrição $(6)$.

A composição corporal foi medida por meio da absorciometria radiológica de energia dupla (DXA) utilizando-se o aparelho Prodigy, GE. A quantidade de massa magra e massa gorda do organismo foi medida em corpo total e membros. A sarcopenia foi aferida pela fórmula de Baumgartner: índice de massa muscular 
$(\mathrm{IMM})=$ somatório da massa magra de membros superiores e inferiores ( $\mathrm{em} \mathrm{kg}$ ) dividido pelo quadrado da altura (em metros). IMM $<5,45 \mathrm{~kg} / \mathrm{m}^{2}$ para mulheres configura sarcopenia (7).

A densidade mineral óssea em coluna lombar, colo do fềmur, fềmur total e corpo total da paciente foi comparada ao banco de dados de pessoas jovens do mesmo sexo (T-score). Conforme critérios da ISCD e SBDens, o mais baixo valor de T-score de cada paciente permitiu classificá-los em: DMO normal ( $\mathrm{T} \geq-1)$, osteopenia $(-2,5<$ T-score $<-1)$ e osteoporose $(\mathrm{T} \leq-2,5)(8)$.

\section{Análise estatística}

Para a análise descritiva das variáveis numéricas contínuas, foram calculadas as médias com os respectivos desvios-padrões. As variáveis qualitativas foram apresentadas por meio de frequências absoluta e relativa. Para verificar a distribuição das variáveis, foi utilizado o teste de Kolmogorov-Smirnov. As correlações entre as variáveis foram verificadas por meio do cálculo do Coeficiente de Correlação de Pearson. As comparações dos níveis de PTH e de filtração glomerular de acordo com a classificação da densidade mineral óssea foram feitas mediante o cálculo do teste Kruskall-Wallis, e as diferenças entre os grupos foram verificadas pelo cálculo do teste de Mann-Whitney com correção de Bonferroni. O nível de significância estatística adotado foi de $5 \%(\mathrm{p}<0,05)$, e todas as análises foram realizadas no pacote estatístico Statistical Package for Social Sciences (SPSS) for Windows versão 13.0.

\section{RESULTADOS}

A média de idade do grupo foi de 78,27 $\pm 6,74$ anos; $86 \%$ eram caucasianas. As principais doenças associadas foram cardiovascular $(88 \%)$, dislipidemia $(50 \%)$, ligadas ao tubo digestivo (38\%), depressão e ansiedade (30\%) e diabetes melito $(28 \%) ; 26 \%$ apresentavam dor crôni- ca. Cinco apresentavam hipotireoidismo compensado, quatro tinham diagnóstico de Alzheimer, mas apresentavam quadro demencial leve, e dois com histórico de câncer, mas que não apresentavam doença ativa no momento da avaliação. O número de medicamentos de uso contínuo foi de $4,77 \pm 1,89$, e metade das idosas $(\mathrm{n}=22)$ utilizava polifarmácia ( $\geq 5$ medicamentos) (9). Nenhuma paciente estava usando glicocorticoide ou anticonvulsivante.

Identificamos baixo peso em $10 \%$ das idosas, eutrofia em $36 \%$, sobrepeso em $28 \%$ e obesidade em $26 \%$. A pontuação média do $\mathrm{MAN}$ foi $25,06 \pm 2,91$. A maioria foi considerada sem risco nutricional $(\mathrm{n}=33)$; dez pacientes apresentavam risco nutricional e uma foi considerada desnutrida.

A composição corporal mostrou $24,95 \pm 8,37 \mathrm{~kg}$ $(39,7 \pm 8,1 \%)$ de massa gorda, enquanto a massa magra foi de 34,31 $\pm 4,90 \mathrm{~kg}$. Pelos critérios de Baumgartner, sete foram consideradas sarcopênicas. Duas idosas apresentaram circunferência braquial inferior a $22 \mathrm{~cm}$ e sete apresentaram circunferência de panturrilha menor que $31 \mathrm{~cm}$. Relações entre idade, fatores nutricionais e sarcopenia constam da tabela 1.

Vinte idosas $(45,5 \%)$ apresentavam função renal adequada ( $\left.\mathrm{FG} \geq 60 \mathrm{ml} / \mathrm{min} / 1,73 \mathrm{~m}^{2}\right) ; 22(50 \%)$ tinham DRC grau 3 (15 na fase $3 \mathrm{~A}$ e 7 na fase $3 \mathrm{~B}$ ), uma $(2,3 \%)$ tinha DRC grau 4 e uma $(2,3 \%)$ estava no grau 5. Detectamos hiperparatireoidismo normocalcêmico em nove das 31 pacientes que dosaram PTH (29\%). A filtração glomerular não se correlacionou com o PTH $(\mathrm{r}=-0,306 ; p=0,094)$ nem com o IMM $(\mathrm{r}=$ $0,197 ; p=0,199)$.

$\mathrm{O}$ envelhecimento influenciou negativamente a função renal $(\mathrm{r}=-0,472 ; p=0,001)$, o IMM $(\mathrm{r}=-0,396$; $p=0,008)$ e parâmetros nutricionais. As circunferências braquial e da panturrilha foram preditores do estado nutricional e da sarcopenia. Esses dados constam da tabela 1 .

Tabela 1. Fatores associados à idade, ao grau de nutrição aferido pelo MNA e à sarcopenia aferida pelo índice IMM de Baumgartner

\begin{tabular}{lccc}
\hline & Idade & MNA & IMM \\
\hline IMC $\left(\mathrm{kg} / \mathrm{m}^{2}\right)$ & $r=-0,319 ; \boldsymbol{p}=\mathbf{0 , 0 3 5}$ & $r=0,408 ; \boldsymbol{p}=\mathbf{0 , 0 0 6}$ & $r=0,719 ; \boldsymbol{p}=\mathbf{0 , 0 0 0}$ \\
C panturrilha (cm) & $r=-0,384 ; \boldsymbol{p}=\mathbf{0 , 0 1 0}$ & $r=0,632 ; \boldsymbol{p}=\mathbf{0 , 0 0 0}$ & $r=0,678 ; \boldsymbol{p}=\mathbf{0 , 0 0 0}$ \\
C braquial (cm) & $r=-0,288 ; p=0,058$ & $r=0,306 ; \boldsymbol{p}=\mathbf{0 , 0 4 4}$ & $r=0,597 ; \boldsymbol{p}=\mathbf{0 , 0 0 0}$ \\
Massa gorda $(\mathrm{kg})$ & $r=-0,334 ; \boldsymbol{p}=\mathbf{0 , 0 2 7}$ & $r=0,352 ; \boldsymbol{p}=\mathbf{0 , 0 1 9}$ & $r=0,576 ; \boldsymbol{p}=\mathbf{0 , 0 0 0}$ \\
Massa magra (kg) & $r=-0,194 ; p=0,206$ & $r=0,104 ; p=0,503$ & $r=0,815 ; \boldsymbol{p}=\mathbf{0 , 0 0 0}$ \\
MNA & $r=-0,077 ; p=0,619$ & - & $r=0,271 ; p=0,075$ \\
\hline
\end{tabular}

IMC: índice de massa corporal; MNA: miniavaliação nutricional; IMM: índice de massa muscular; $r$ : coeficiente de correlação de Pearson; $p$ estatisticamente significativo quando $<0,05$. 
Com base no mais baixo valor de T-score obtido por meio da densitometria, $34,1 \%$ tinham osteoporose, $52,3 \%$ osteopenia e $13,6 \%$ DMO normal. Não foi realizada radiografia da coluna toracolombar para busca ativa de fraturas vertebrais não clínicas, mas havia história de fratura prévia (Colles, vértebras, clavícula) em onze. Entre essas fraturadas, somente quatro mostraram densitometria compatível com osteoporose (e usavam alendronato); cinco foram classificadas como osteopenia e duas como DMO normal. Entretanto, não foi possível excluir uso pregresso de bisfosfonatos.

A massa óssea não se correlacionou com a idade e a filtração glomerular, não havendo diferenças nos níveis médios de FG corr. $\left(\mathrm{ml} / \mathrm{min} / 1,73 \mathrm{~m}^{2}\right)$ entre os subgrupos classificados como osteoporose $(52,50 \pm$ $13,38)$, osteopenia $(62,33 \pm 20,90)$ e DMO normal $(60,87 \pm 19,02), p=0,34$. O PTH mostrou-se inversamente associado ao T-score no fềmur, mas não foram identificadas diferenças nos níveis médios do PTH (pg/ $\mathrm{mL}$ ) entre pacientes classificadas como osteoporose $(66,53 \pm 93,14 ; \mathrm{n}=12)$, osteopenia $(70,02 \pm 52,69 ; \mathrm{n}$ $=15)$ e DMO normal $(29,43 \pm 20,9 ; \mathrm{n}=4) p=0,15$. Por outro lado, o estado nutricional e o IMM tiveram grande influência na massa óssea. Essas análises constam da tabela 2 .

\section{DISCUSSÃO}

A população feminina predomina no ambulatório de Geriatria: as mulheres são mais longevas e cuidam mais da saúde. A maioria das idosas estudadas apresentava excesso de peso para a idade, provavelmente por mudanças no padrão alimentar, na composição dos alimentos e pelo sedentarismo $(10,11)$.
Doenças cardiovasculares foram as comorbidades mais prevalentes. Segundo os resultados do Framingham Heart Study (1948) e do INTERHEART Study (1999), fatores que aumentam o risco de doenças cardiovasculares são hipertensão arterial, dislipidemia, obesidade, diabetes melito e hábitos inadequados, como dietas hipercalóricas, gorduras saturadas, sódio, tabagismo, etilismo e sedentarismo (12). Dislipidemia foi frequente em nosso estudo, embora houvesse poucos casos de diabetes melito se compararmos com a literatura (13-15). Além dos fatores de risco cardiovasculares, preocupa o crescente diagnóstico de depressão com o envelhecimento; debilidade física, alterações do sono e perda do cônjuge contribuem para o quadro da doença, bem como risco para demência e institucionalização (11).

Por meio da MAN, constatamos poucos idosos com desnutrição e a grande maioria não tinha risco nutricional. Em estudo em que foram feitas avaliações nutricionais e acompanhamento regular de 178 idosos, apenas $3 \%$ apresentaram desnutrição e $48 \%$, risco de desnutrição segundo a MAN (17).

Sarcopenia é uma consequência importante do envelhecimento, tendo como características a perda de massa muscular, associada à redução na força e potência muscular. Entre 20 e 80 anos de idade, o declínio cumulativo na massa de músculos esqueléticos é da ordem de $35 \%$ a $40 \%$, em decorrência da perda de fibras musculares e da atrofia seletiva das fibras do tipo II. A redução de força muscular é ainda maior depois dos 70 anos; $28 \%$ dos homens com 74 anos não conseguem levantar sobre suas cabeças objetos com peso maior que 25 quilos. Com o aumento da idade, há uma redução progressiva na potência muscular, na velocidade da ge-

Tabela 2. Fatores associados à densidade mineral óssea

\begin{tabular}{|c|c|c|c|c|}
\hline & Mais baixo T-score & T-score coluna lombar & T-score colo fêmur & T-score fêmur total \\
\hline Idade (anos) & $r=-0,175, p=0,257$ & $r=0,002, p=0,991$ & $r=-0,193, p=0,210$ & $r=-0,261, p=0,086$ \\
\hline $\mathrm{FG}$ corr (ml/min/1,73 m²) & $r=0,224, p=0,143$ & $r=0,026, p=0,868$ & $r=0,335, \boldsymbol{p}=\mathbf{0 , 0 2 6}$ & $r=0,353, \boldsymbol{p}=\mathbf{0 , 0 1 9}$ \\
\hline Log PTH (pg/mL) & $r=-0,021, p=0,910$ & $r=-0,069, p=0,712$ & $r=-0,143, p=0,442$ & $r=-0,131, p=0,483$ \\
\hline IMC $\left(\mathrm{kg} / \mathrm{m}^{2}\right)$ & $r=0,274, \boldsymbol{p}=\mathbf{0 , 0 7 2}$ & $r=0,338, \boldsymbol{p}=\mathbf{0 , 0 2 5}$ & $r=0,047, p=0,761$ & $r=0,215, p=0,161$ \\
\hline MAN & $r=0,378, \boldsymbol{p}=\mathbf{0 , 0 1 1}$ & $r=0,350, \boldsymbol{p}=\mathbf{0 , 0 2 0}$ & $r=0,198, p=0,198$ & $r=0,254, \boldsymbol{p}=\mathbf{0 , 0 9 6}$ \\
\hline C panturrilha (cm) & $r=0,213, p=0,165$ & $r=0,201, p=0,191$ & $r=0,049, p=0,754$ & $r=0,168, p=0,276$ \\
\hline C braquial (cm) & $r=0,237, p=0,121$ & $r=0,297, \boldsymbol{p}=\mathbf{0 , 0 5 0}$ & $r=0,002, p=0,989$ & $r=0,194, p=0,207$ \\
\hline Massa gorda (kg) & $r=0,305, \boldsymbol{p}=\mathbf{0 , 0 4 4}$ & $r=0,349, \boldsymbol{p}=\mathbf{0 , 0 2 0}$ & $r=0,099, p=0,522$ & $r=0,244, p=0,110$ \\
\hline Massa magra (kg) & $r=0,253, \boldsymbol{p}=\mathbf{0 , 0 9 8}$ & $r=0,324, \boldsymbol{p}=\mathbf{0 , 0 3 2}$ & $r=0,086, p=0,579$ & $r=0,245, p=0,110$ \\
\hline IMM (kg/m²) & $r=0,258, \boldsymbol{p}=\mathbf{0 , 0 9 1}$ & $r=0,228, \boldsymbol{p}=\mathbf{0 , 1 3 7}$ & $r=0,152, p=0,325$ & $r=0,324, p=0,032$ \\
\hline
\end{tabular}

IMC: índice de massa corporal; MNA: miniavaliação nutricional; IMM: índice de massa muscular; r: coeficiente de correlação de Pearson; $p$ estatisticamente significativo quando < 0,05. 
ração de força e na resistência à fadiga, que acabam diminuindo a capacidade de persistir em uma tarefa. A perda de força e massa muscular leva a uma deterioração da função física, como indicado pela prejudicada habilidade de levantar de uma cadeira, subir degraus, acelerar o passo e manter o equilíbrio. A deterioração da função física contribui para a perda da independência, para a depressão e para o aumento do risco de quedas e fraturas no idoso. A perda de massa muscular não costuma resultar em perda de peso, devido à natural substituição por gordura corporal (18-20). Em nosso estudo, apenas $10 \%$ das idosas foram consideradas sarcopênicas, a despeito do elevado número de comorbidades; uma explicação pode ser a população analisada: idosas adequadamente nutridas ou com excesso de peso e capazes de frequentar o ambulatório de Geriatria. De importância prática, destacamos que massa gorda, circunferência braquial e da panturrilha associaram-se ao índice de Baumgartner como preditores da sarcopenia; esse achado foi de grande relevância clínica.

A redução progressiva da filtração glomerular foi outra característica do envelhecimento, descrita na literatura (2l) e evidenciada em nosso estudo, que colabora para o catabolismo proteico e a piora da saúde geral. Certamente comorbidades como hipertensão arterial e diabetes melito contribuíram para esse declínio. Menos da metade das idosas estudadas tinham função renal adequada, mas isso não se refletiu no desencadeamento da sarcopenia, talvez por não estarem na fase final de DRC como é o caso de outras populações (22). Outras consequências esperadas do declínio da FG são hiperparatireoidismo secundário e aumento da reabsorção esquelética, favorecendo o surgimento de osteoporose (23). Em nossa casuística, 29\% das pacientes apresentaram hiperparatireoidismo normocalcêmico, mas a elevação do PTH não estava associada ao declínio da FG. Não analisamos a 25-hidroxivitamina D dessas pacientes, mas é possível que a deficiência de vitamina $\mathrm{D}$, muito frequente inclusive em brasileiros (24-26), seja a maior causa do hiperparatireoidismo.

$\mathrm{O}$ diagnóstico de osteoporose à densitometria foi feito em proporção semelhante comparando-se coluna lombar e fêmur proximal. Pela densitometria, apenas um terço das idosas teria osteoporose, mas a fragilidade óssea certamente foi subestimada, haja vista pacientes com fratura osteoporótica clínica cuja densitometria mostrava "osteopenia" ou mesmo "DMO normal" isso sem falar que não pesquisamos ativamente fraturas vertebrais não clínicas, que correspondem a mais de
$50 \%$ das fraturas vertebrais (27). A literatura reconhece amplamente a existência de fraturas em pessoas idosas não osteoporóticas à densitometria $(28,29)$, e isso decorre basicamente das limitações do próprio método, que afere quantidade óssea, mas não a qualidade óssea. Entretanto, não podemos descartar o uso prévio de bisfosfonatos, promovendo melhora da DMO e mudança na classificação de osteoporose para osteopenia ou normalidade.

O PTH não se associou com a presença de osteoporose, nem se correlacionou com os valores do T-score obtidos à densitometria óssea. Curiosamente, o declínio da FG contribuiu significativamente para a redução da DMO apenas no fêmur proximal, sítio onde predomina osso cortical, mas esse efeito aparentemente não foi mediado pelo hiperparatireoidismo.

Nem mesmo a idade teve impacto direto na massa óssea, que foi mais influenciada pelo estado nutricional, aferido pelo IMC e MAN, pela massa gorda e a sarcopenia, esta última estimada pela massa magra.

Reconhecemos que o grupo estudado foi pequeno, mas permitiu concluirmos que fatores nutricionais e composição corporal (índice de massa muscular) são importantes preditores da saúde óssea.

\section{CONCLUSÕES}

Poucos idosos apresentavam risco nutricional e/ou sarcopenia. Entretanto, as grandes influências na massa óssea foram o estado nutricional, a massa gorda e a sarcopenia aferida pelo índice de massa muscular. Isso nos leva a enfatizar a importância de boa nutrição e exercícios físicos adequados à idade para a população de idosos ambulatoriais.

Agradecimentos: aos colegas do Ambulatório de Geriatria, aos técnicos da Densitometria e aos pacientes que participaram da pesquisa.

Declaração de financiamento: este trabalho contou com bolsa de apoio do Conselho Nacional de Desenvolvimento Científico e Tecnológico $(\mathrm{CNPq})$, mediante a bolsa de mestrado a mim concedida.

Declaração: os autores declaram não haver conflitos de interesse científico neste estudo.

\section{REFERÊNCIAS}

1. Pereira RS, Curioni CC, Veras R. Perfil demográfico da população idosa no Brasil e no Rio de Janeiro em 2002. Textos Envelhecimento [online] 2003;6(1):43-59. 
2. Acuña K, Cruz T. Avaliação do estado nutricional de adultos e idosos e situação nutricional da população brasileira. Arq Bras Endocrinol Metab. 2004;48(3):345-61.

3. Prigent A. Monitoring renal function and limitations of renal function tests. Sem Nucl Med. 2008;38:32-46.

4. Kidney Disease: Improving Global Outcomes (KDIGO) CKD-MBD Work Group. KDIGO clinical practice guideline for the diagnosis, evaluation, prevention, and treatment of chronic kidney disease-mineral and bone disorder (CKD-MBD). Kidney Int. 2009;76 (Suppl 113): S1-30.

5. Moreira AJ, Nicastro H, Cordeiro RC, Coimbra P, Frangella VS. Composição corporal de idosos segundo a antropometria. Rev Bras Geriatr Gerontol. 2009;12(2):201-13.

6. EmedTCXS, Kronbauer A, Magnoni D. Mini-avaliação nutricional como indicador de diagnóstico em idosos de asilos. Rev Bras Nutr Clin. 2006;21(3):219-23.

7. Pierine DT, Nicola M, Oliveira EP. Sarcopenia: alterações metabólicas e consequências no envelhecimento. R Bras Ci Mov 2009;17(3):96-103.

8. Brandão CMA, Camargos BM, Zerbini CA, Zerbini CA, Plapler PG, Mendonça LMC, et al. Posições oficiais 2008 da Sociedade Brasileira de Densitometria Óssea (SBDens). Arq Bras Endocrinol Metab. 2009;53(1):107-12.

9. Secoli SR. Polifarmácia: interações e reações adversas no uso de medicamentos por idosos. Rev Bras Enferm. 2010;63(1):136-40.

10. Amado TCF, Arruda IKG, Ferreira RAR. Aspectos alimentares, nutricionais e de saúde de idosas atendidas no Núcleo de Atenção ao Idoso - NAI, Recife/2005. ALAN SLAN. 2007;57(4):366-72.

11. Cabrera MAS, Jacob Filho W. Obesidade em idosos: prevalência, distribuição e associação com hábitos e co-morbidades. Arq Bras Endocrinol Metab. 2001;45(5):494-501.

12. Ferreira CCC, Peixoto MRG, Barbosa MA, Silveira EA. Prevalência de fatores de risco cardiovascular em idosos usuários do Sistema Único de Saúde de Goiânia. Arq Bras Cardiol. 2010;95(5):621-8.

13. Bortoli C, Bonatto S, Bruscato NM, Siviero J. Ingestão dietética de gordura saturada e carboidratos em adultos e idosos com dislipidemias oriundos do Projeto Veranópolis. Rev Bras Cardiol. $2011 ; 24(1): 33-41$

14. Alves LC, Leimann BCQ, Vasconcelos MEL, Carvalho MS, Vasconcelos AGG, Fonseca TCO, et al. A influência das doenças crônicas na capacidade funcional dos idosos do Município de São Paulo, Brasil. Cad Saúde Pública. 2007;23(8):1924-30.

15. Sampaio LR. Avaliação nutricional e envelhecimento. Rev Nutr. 2004;17(4):507-14

16. Sousa VMC, Guariento ME. Avaliação do idoso desnutrido. Rev Bras Clin Med. 2009;(7):46-9.

17. Castro PR, Frank AA. Mini-avaliação nutricional na determinação do estado de saúde de idosos com ou sem a doença de Alzhei- mer: aspectos positivos e negativos. Estud Interdiscipl Envelhec. 2009;14(1):45-64.

18. SilvaTAA, Frisoli Junior A, Pinheiro MM, Szejnfeld VL. Sarcopenia associada ao envelhecimento: aspectos etiológicos e opções terapêuticas. Rev Bras Reumatol. 2006;46(6):391-7.

19. Leite LEA, Resende TL, Nogueira GM, Cruz IBM, Schneider RH, Gottlieb MGV. Envelhecimento, estresse oxidativo e sarcopenia: uma abordagem sistêmica. Rev Bras Geriatr Gerontol. 2012;15(2):365-80.

20. Teixeira VON, Filippin LI, Xavier RM. Mecanismos de perda muscular da sarcopenia. Rev Bras Reumatol. 2012;52(2):247-59.

21. Glassock RJ, Rule AD. The implications of anatomical and functional changes of the aging kidney: with an emphasis on the glomeruli. Kidney Int. 2912;82:270-7.

22. Avesani CM, Carrero JJ, Axelsson J, Qureshi AR, Lindholm B, Stenvinkel P. Inflammation and wasting in chronic kidney disease: partners in crime. Kidney Int. 2006;70:S8-13.

23. Craver L, Marco MP, Martínez I, Rue M, Borràs M, Martín ML, et al. Mineral metabolism parameters throughout chronic kidney disease stages 1-5. Achievement of K/DOQI target ranges. Nephrol Dial Transplant. 2007;22:1171-6.

24. Saraiva GL, Cendoroglo MS, Ramos LR, Araújo LMQ, Vieira JGH, Maeda SS, et al. Prevalência da deficiência, insuficiência de vitamina D e hiperparatireoidismo secundário em idosos institucionalizados e moradores na comunidade da cidade de São Paulo, Brasil. Arq Bras Endocrinol Metabol. 2007;51:437-42.

25. Silva BCC, Camargos BM, Fujii JB, Dias EP, Soares MMS. Prevalência de deficiência e insuficiência de vitamina $D$ e sua correlação com PTH, marcadores de remodelação óssea e densidade mineral óssea, em pacientes ambulatoriais. Arq Bras Endocrinol Metab. 2008;52(3):482-8.

26. Bandeira F, Griz L, Freese E, Lima DC, Thé AC, Diniz ET. Vitamin D deficiency and its relationship with bone mineral density among postmenopausal women living in the tropics. Arq Bras Endocrinol Metab. 2010;54(2):227-32.

27. Fink HA, Milavetz DL, Palermo L, Nevitt MC, Cauley JA, Genant $\mathrm{HK}$, et al. What proportion of incident radiographic vertebral deformities is clinically diagnosed and vice versa? J Bone Miner Res. 2005;20:1216-22.

28. Schuit SCE, Van der Klift, Weel AEAM, de Laet CEDH, Burger $\mathrm{H}$, Seeman $E$, et al. Fracture incidence and association with bone mineral density in elderly men and women: the Rotterdam Study. Bone. 2004;34:195-202.

29. Siris ES, Brenneman SK, Barrett-Connor E, Miller PD, Sajjan S, Berger $\mathrm{ML}$, et al. The effect of age and bone mineral density on the absolute, excess, and relative risk of fracture in postmenopausal women aged 50-99: results from the National Osteoporosis Risk Assessment (NORA) Osteoporos Int. 2006;17:565-74. 